\title{
Using reduced rank regression methods to identify dietary patterns associated with obesity: a cross-country study among European and Australian adolescents
}

Inge Huybrechts ${ }^{1 *}+$ Sandrine Lioret $^{2,3,4} \dagger$ Theodora Mouratidou ${ }^{5}$, Marc J. Gunter ${ }^{1}$, Yannis Manios ${ }^{6}$, Mathilde Kersting ${ }^{7}$, Frederic Gottrand ${ }^{8}$, Anthony Kafatos ${ }^{9}$, Stefaan De Henauw ${ }^{10}$, Magdalena Cuenca-García $^{11}$, Kurt Widhalm ${ }^{12}$, Marcela Gonzales-Gross ${ }^{13,14,15}$, Denes Molnar ${ }^{16}$, Luis A. Moreno ${ }^{5}$ and Sarah A. McNaughton ${ }^{4}$

${ }^{1}$ International Agency for Research on Cancer, Nutrition and Metabolism Section, 69372 Lyon CEDEX 08, France

${ }^{2}$ Centre for Physical Activity and Nutrition Research, School of Exercise and Nutrition Sciences, Deakin University, Melbourne, VIC 3125, Australia

${ }^{3}$ Inserm, UMR1153 Epidemiology and Statistics Sorbonne Paris Cité Research Center (CRESS), Early ORigins of Child Health And Development Team (ORCHAD), Villejuif, F-94807, France

${ }^{4}$ Paris-Descartes University, Paris, F-75014, France

${ }^{5}$ Growth, Exercise, Nutrition and Development (GENUD) Research Group, School of Health Science (EUCS), University of Zaragoza, C/ Domingo Miral s/n, 50009 Zaragoza, Spain

${ }^{6}$ Department of Nutrition and Dietetics, School of Health Science and Education, Harokopio University, Athens 17671, Greece

${ }^{7}$ Research Institute of Child Nutrition, Rheinische Friedrich-Wilhelms-University Bonn, 44225 Dortmund, Germany

${ }^{8}$ UMR995, Faculty of Medicine, University of Lille, CHRU, 59045 Lille, France

${ }^{9}$ Preventive Medicine and Nutrition Unit, School of Medicine, University of Crete, PO Box 2208 GR-71003, Heraklion, Crete, Greece

${ }^{10}$ Department of Public Health, Faculty of Medicine and Health Sciences, Ghent University, Ghent 9000, Belgium

${ }^{11}$ Department of Medical Physiology, School of Medicine, University of Granada, 18014 Granada, Spain

${ }^{12}$ Department of Pediatrics, Private Medical University, 5020 Salzburg, Austria

${ }^{13}$ ImFINE Research Group, Department of Health and Human Performance, Faculty of Physical Activity and Sport Sciences (INEF), Technical University of Madrid, Madrid E-28040, Spain

${ }^{14}$ Department of Nutrition and Food Science, University of Bonn, D-53115 Bonn, Germany

${ }^{15}$ CIBER: CB12/03/30038 Fisiopatología de la Obesidad y la Nutrición, CIBERobn, Instituto de Salud Carlos III (ISCIII), 28029 Madrid, Spain

${ }^{16}$ Department of Paediatrics, Medical Faculty, H-7624 Pécs, Hungary

(Submitted 18 August 2016 - Final revision received 7 December 2016 - Accepted 21 December 2016 - First published online 7 Febraury 2017)

\section{Abstract}

This study aims to examine repeatability of reduced rank regression (RRR) methods in calculating dietary patterns (DP) and cross-sectional associations with overweight (OW)/obesity across European and Australian samples of adolescents. Data from two cross-sectional surveys in Europe (2006/2007 Healthy Lifestyle in Europe by Nutrition in Adolescence study, including 1954 adolescents, 12-17 years) and Australia (2007 National Children's Nutrition and Physical Activity Survey, including 1498 adolescents, 12-16 years) were used. Dietary intake was measured using two non-consecutive, 24-h recalls. RRR was used to identify DP using dietary energy density, fibre density and percentage of energy intake from fat as the intermediate variables. Associations between DP scores and body mass/fat were examined using multivariable linear and logistic regression as appropriate, stratified by sex. The first DP extracted (labelled 'energy dense, high fat, low fibre') explained 47 and $31 \%$ of the response variation in Australian and European adolescents, respectively. It was similar for European and Australian adolescents and characterised by higher consumption of biscuits/cakes, chocolate/confectionery, crisps/savoury snacks, sugar-sweetened beverages, and lower consumption of yogurt, high-fibre bread, vegetables and fresh fruit. DP scores were inversely associated with BMI $z$-scores in Australian adolescent boys and borderline

Abbreviations: \%BF, body fat percentage; ALSPAC, Avon Longitudinal Study of Parents and Children; DED, dietary energy density; DP, dietary pattern; FD, fibre density; HELENA, Healthy Lifestyle in Europe by Nutrition in Adolescence study; MVPA, moderate to vigorous physical activity; NCNPAS, National Children's Nutrition and Physical Activity Survey; OW, overweight; PA, physical activity; RRR, reduced rank regression; SB, sedentary behaviour; SES, socio-economic status.

* Corresponding author: I. Huybrechts, fax +3347273 8361, email huybrechtsi@iarc.fr

$\dagger$ These authors contributed equally to this work. 
inverse in European adolescent boys (so as with \%BF). Similarly, a lower likelihood for OW in boys was observed with higher DP scores in both surveys. No such relationships were observed in adolescent girls. In conclusion, the DP identified in this cross-country study was comparable for European and Australian adolescents, demonstrating robustness of the RRR method in calculating DP among populations. However, longitudinal designs are more relevant when studying diet-obesity associations, to prevent reverse causality.

Key words: Dietary patterns: Adolescents: Reduced rank regression analysis: Healthy Lifestyle in Europe by Nutrition in Adolescence study: Australian National Children's Nutrition and Physical Activity Survey

Obesity is a major public health concern in both developed and developing countries ${ }^{(1)}$ with nutrition-related factors being one of the main determinants ${ }^{(2)}$. Individual foods and nutrients are part of the overall diet, with some arguing that this is one of the reasons why studies assessing diet-diseases associations, on the basis of individual foods and/or nutrients, fail to identify strong associations as the impact of many other dietary factors might be neglected ${ }^{(3)}$. Instead, studying dietary patterns (DP) is suggested as a complementary and more comprehensive and powerful method to investigate such associations ${ }^{(4-7)}$.

There are a number of methods to derive DP, namely the development of diet quality scores or indices based on a priori knowledge of dietary guidelines or the use of multivariate statistical methods ${ }^{(8)}$. These statistical methods are a posteriori data reduction techniques, which summarise the variation in food intakes into a small number of patterns or clusters. Principal component analysis and cluster analysis are commonly used techniques to derive patterns and were shown to have good reproducibility across studies ${ }^{(9)}$. However, a newer method called reduced rank regression (RRR) is increasingly used in studies ${ }^{(4-7,10-13)}$. The RRR technique is often considered a hybrid method as it combines multivariate approaches with existing knowledge of diet-disease associations, and may provide novel understanding of the pathways through which diet influences disease.

RRR has been used with a wide range of health outcomes and intermediate markers in adults ${ }^{(4-7,14-19)}$. However, its use in adolescents is less widespread $^{(11-13)}$. Furthermore, a significant methodological issue facing dietary pattern research is reproducibility of DP across cohorts when such data-driven techniques are used. Few studies have, however, attempted to replicate the findings of other studies and investigate previously identified RRR DP ${ }^{(11,12,20,21)}$.

Using data from 5- to 9-year-old children in the Avon Longitudinal Study of Parents and Children (ALSPAC), Johnson et al. ${ }^{(13)}$ identified a dietary pattern that predicted dietary energy density (DED), fibre density (FD) and percentage of energy intake from fat (used as intermediate marker in the RRR analysis) and demonstrated that this was prospectively associated with fat mass (FM) and adiposity. This 'energy-dense, high-fat, low-fibre' dietary pattern was replicated at 7,10 and 13 years in a subsequent study on the basis of same data set ${ }^{(20)}$, showing similar, though attenuated longitudinal associations with adiposity measured up to 15 years of age. Appannah et al. ${ }^{(11,12)}$ obtained a similar dietary pattern using the same RRR methodology in adolescents at 14 and 17 years of age from the Raine Study, a population-based cohort recruited in Perth, Western Australia, suggesting some reproducibility across these two cohorts.
The aim of this study was to further examine the repeatability of RRR methods across European and Australian samples of adolescents, using the Healthy Lifestyle in Europe by Nutrition in Adolescence study (HELENA) and the Australian National Children's Nutrition and Physical Activity Survey (NCNPAS), both collected in 2007. In addition, we tested a positive crosssectional relationship hypothesised between the dietary pattern and obesity risk in the European and Australian samples of adolescents.

\section{Methods}

\section{Study population}

This study is based on the analysis of two cross-sectional surveys performed in 2007 among European (HELENA) and Australian (NCNPAS) adolescents, using similar dietary assessment method.

Healthy Lifestyle in Europe by Nutrition in Adolescence study. The HELENA study is a multicentre study focusing on lifestyle habits and nutritional status by region, cultural background, socio-economic status (SES), age and sex among European adolescents (12.5-17.5 years old) from ten European cities (Athens, Heraklion, Dortmund, Ghent, Lille, Pecs, Rome, Stockholm, Vienna and Zaragoza), and was conducted between 2006 and 2007. A random cluster sampling (all pupils, from a random selection of classes, from all schools in the ten European cities) of 3528 adolescents, stratified by geographical location, age and SES, was carried out. Because of logistical reasons, data from Heraklion and Pecs were not included in the dietary intake analysis ( $7 \%$ of the total sample). Considering all the variables included in the models used in these analyses, data were available from 1954 participants aged 12.5-17.5 years. The study was approved by the Research Ethics Committees of each city involved. More details on operational and sampling procedures have been published elsewhere ${ }^{(22)}$.

Dietary intake assessment. Dietary intake was assessed using the self-administered, computerised 24-h recalls HELENADietary Assessment Tool (DIAT) on the basis of Young Adolescents' Nutrition Assessment software (YANA-C). The validity of the tool in European adolescents for all nutrients and energy intakes, expressed as Spearman correlations, ranged from $r \mathrm{~s}$ $0 \cdot 86$ to $0 \cdot 91^{(23,24)}$. The adolescents completed the 24 -h recalls twice $^{(25)}$ during school time and within a time span of 2 weeks; both times, trained dietitians were present. To calculate energy and nutrient intakes, data of the HELENA-DIAT were linked to 
the German Food Code and Nutrient Database (Bundeslebensmittelschlussel (BLS) version II.3.1, 2005). For this purpose, culture-specific composite dishes were disaggregated into their basic food components, all of which were available in the German database.

Physical activity and sedentary behaviour assessments. Patterns of physical activity (PA) were assessed using the International Physical Activity Questionnaire for Adolescents (IPAQ-A) ${ }^{(26,27)}$. Data were cleaned and truncated following the guidelines provided by the IPAQ group (www.ipaq.ki.se) ${ }^{(26,27)}$. Variables obtained by the IPAQ-A for the current study were the time spent (min/week) in moderate PA, vigorous PA and moderate to vigorous physical activity (MVPA). Only the MVPA indicator was used in this paper. A binary indicator 'meeting PA recommendations' was created by dividing those who comply with the WHO recommendation of $60 \mathrm{~min} \mathrm{MVPA} / \mathrm{d} v$. those who do not comply with this recommendation ${ }^{(28)}$

Sedentary behaviour (SB) was assessed by a self-reported HELENA questionnaire that was shown to be a reliable tool to be used in adolescents ${ }^{(29)}$. The questionnaire included daily minutes of the following sedentary items: television viewing, playing with computer, playing with console games, use of Internet for non-study reasons and use of Internet for study and studying/homework (lessons not included). The average time spent per day in each of those sedentary activities was calculated. In this analysis, total screen time (the sum of average $\mathrm{min} / \mathrm{d}$ television viewing + playing with computer + playing with console games + use of Internet for non-study reasons + use of Internet for study), divided in quintiles, was used as indicator for screen SB.

Anthropometric measurements. Height, weight and six skinfolds were measured using standard procedures as described elsewhere ${ }^{(30)}$. BMI was calculated by dividing body weight in kilograms by squared body height in metres and then transformed to age- and sex-specific $z$-scores. BMI $z$-scores and overweight (OW) status (OW/obese $v$. thin/normal weight) were defined using age- and sex-specific cut-off values according to Cole et $a l .^{(31,32)}$. Skinfold thickness was measured at the triceps, biceps, subscapular, suprailiac, thigh and calf on the left side of the body using a Holtain Caliper (Holtain Ltd). Body fat percentage (\%BF) and FM were calculated from skinfold thicknesses (triceps and subscapularis) using the equation of Slaughter et al. ${ }^{(33)}$

Socio-economic status. Among the various variables allowing the characterisation of SES in the HELENA study, we used maternal education level, defined in three categories, that is low education, high secondary education and university degree.

\section{Australian National Children's Nutrition and Physical} Activity Survey. The 2007 NCNPAS study aimed to assess food and nutrient intakes, PA participation, and to measure weight and height of a national sample of Australian children and adolescents aged 2-16 years. The sampling, selection and recruitment methodology are described in detail elsewhere ${ }^{(34)}$. Households were selected using random digit dialling of telephone numbers from within clusters based on postcode (stratified by state/territory and capital city/rest of state) and one child from each selected household was invited to participate. A total of 4487 children completed the entire survey with approximately equal representation of boys and girls in each of four age groups (2-3, 4-8, 9-13 and 14-16 years) from February to August 2007. Data were available from 1647 participants aged 12-16 years for this analysis.

Dietary intake assessment. Intake of foods and beverages was assessed using 24-h recall methodology using a computerassisted interviewing system. The three-pass method consisted of the completion of a quick list of food and beverage items consumed, followed by the collection of detailed information for each item listed in the quick recall; and finally a review phase to allow respondents to report any foods that may have been forgotten. A food model booklet was provided to estimate food portion sizes. Each child completed $1 \times 24$-h recall during a home visit (via computer-assisted personal interview (CAPI)) and a second 24-h recall 7-21 days later by telephone (via computer-assisted telephone interview (CATI)). In order to capture intakes and activity patterns that would represent all types of days, the CAPI and the CATI were collected on different day types when possible. Nutrient intake was calculated by using a customised food composition database ${ }^{(35)}$.

Physical activity and sedentary behaviour assessments. PA was measured using a previously validated computerised 24-h recall known as the Multimedia Activity Recall for Children and Adolescents $^{(36)}$. Each child recalled $4 \mathrm{~d}$ worth of activity, $2 \mathrm{~d}$ before the CAPI and $2 \mathrm{~d}$ before the CATI. Children reported everything they did in the previous $48 \mathrm{~h}$. Activity data collected as part of the children's survey include each child's PA level and the number of minutes per day spent in the following categories: MVPA, vigorous activity level, organised sport and play, free play, active transport, out of school hours screen time, television, video games, computer use, passive transport, nonscreen SB and sleep. The number of minutes spent in these activities was calculated for each child on each of the 4 sampled days. Consistent with the variables used from the HELENA study, the average daily number of minutes children spent in MVPA (treated as binary, meeting the recommendation of $60 \mathrm{~min} \mathrm{MVPA} / \mathrm{d}$ ) and average screen time (the sum of television, video games and computer times), divided in quintiles, were used in this analysis.

Anthropometric measurements. Weight and height were measured for all participants during the home visit ${ }^{(37)}$. BMI $z$-scores were calculated and categorised in the same way as outlined for HELENA ${ }^{(32)}$.

Socio-economic status. Socio-demographic information about the households of the participants included in the children's survey was collected during the home visit, including information about the children and the parents or up to two caregivers. Consistent with the definition used in the HELENA study, maternal education (low education, high secondary education and university degree) was used as a proxy of SES.

Both the HELENA study and the Australian NCNPAS survey were conducted according to the guidelines laid down in the Declaration of Helsinki. 


\section{Statistical analysis}

Harmonisation and preparation of dietary data in Healthy Lifestyle in Europe by Nutrition in Adolescence study and National Children's Nutrition and Physical Activity Survey. Food intake data were collapsed into forty-seven food groups according to usage or differences in energy density and fat and fibre contents as described for the ALSPAC study ${ }^{(13)}$ with the addition of alcohol for these adolescent samples. Average absolute intakes $(\mathrm{g} / \mathrm{d})$ were computed for each food group. Consistent with the ALSPAC study ${ }^{(13)}$, we estimated total energy intake $(\mathrm{kJ} / \mathrm{d})$, percentage of energy intake from fat, FD $(\mathrm{g} / \mathrm{MJ})$ and DED $(\mathrm{kJ} / \mathrm{g})$. DED was calculated, excluding drinks, by dividing total food energy $(\mathrm{kJ})$ by total food weight $(\mathrm{g})$ and $\mathrm{FD}$ by dividing NSP fibre intake (g) by total EI (MJ).

As under-reporting of energy intake is common, particularly among adolescents ${ }^{(38,39)}$ and in OW populations, underreporters were considered as individuals with a ratio of energy intake over estimated BMR, calculated with the equation of Schofield ${ }^{(40)}$, lower than 0.96 according to the Goldberg cut-offs. The sensitivity and specificity of the Goldberg criterion to identify diet reports of poor validity has been previously tested $^{(41,42)}$. Rather than excluding energy under-reporters, and consistent with Johnson et al. ${ }^{(13)}$, a categorical variable indicating under-reporters $v$. plausible reporters was included as a potential confounder in statistical models.

All lifestyle and socio-demographic variables were harmonised, using the same indicators, as previously described. Extra inclusion criteria for the purpose of the current analysis included: having complete SES, PA and SB variables and having provided two 24-h dietary recalls resulting in 1954 adolescents for HELENA and 1498 for NCNPAS.

'Exploratory' reduced rank regression analysis. DP were determined using RRR in both HELENA and NCNPAS as previously described by Johnson et al. ${ }^{(13)}$. RRR determines factors from food intake data that maximise the explained variation in the intermediate markers that are hypothesised to be related to the health outcome. DED, percentage of energy intake from fat and FD were used as the intermediate markers. RRR analysis was conducted using SAS (version 9; SAS Institute). The number of DP extracted using RRR analysis is determined by the number of intermediate markers/variables. The first pattern explained $47 \%$ for the Australian adolescents and $31 \%$ for the European adolescents of the response variation in each cohort and was used in the subsequent analyses, as per Johnson et al. ${ }^{(13)}$. The cumulated explained variance by patterns 2 and 3 was rather low in both the European and Australian data set $(<22 \%)$ and was therefore not further considered in our analysis. RRR was run in the whole sample and separately in boys and girls. Because the sex-specific patterns were qualitatively the same as those derived from the whole sample, indicated by similar pattern loadings, the factor loadings derived from the whole sample were used in the present analysis (Table 1). Dietary pattern scores were calculated for each adolescent as a linear combination of all food group intakes with factor loadings' absolute value $>0 \cdot 15$. Indeed, to interpret the results and calculate the scores, we retained the items most strongly related to each pattern, as recommended by Schulze et $a l .{ }^{(43)}$, that is those for which the absolute value of the loading coefficient was $>0 \cdot 15$. This threshold was chosen accounting for the overall range of loadings observed in our data (i.e. the ranking of foods in the pattern) and both the interpretability and differentiation of each pattern.

The resulting DP were qualitatively compared between the two studies included in this paper (HELENA and NCNPAS) and also with the identified patterns from the ALSPAC cohort ${ }^{(13)}$. Associations were examined between dietary pattern scores and both BMI $z$-scores and OW using multivariable linear and logistic regression as appropriate. In addition, in HELENA, the association between dietary pattern scores and \%BF was investigated via multivariable linear regression analysis. Analyses were stratified by sex due to observed significant differences in dietary intakes (e.g. energy intakes, etc.) between boys and girls and adjusted for the same covariates in both data sets, namely age, energy under-reporting status, meeting PA recommendations, screen sedentary time and maternal education level. Possible interactions between DP scores and underreporting status were tested. STATA version 12 was used to perform the regression analysis. $P$ values $<0.05$ were considered to be statistically significant.

\section{Results}

\section{Characteristics of the study populations}

This analysis included 1954 participants in the HELENA study ( $45 \%$ boys; mean age of 14.6 (SD 1.2) years) and 1498 participants from the NCNPAS study ( $50 \%$ boys; mean age $14 \cdot 2$ (SD 1.3) years). Table 1 shows the descriptive analysis of the studied variables stratified by sex, in both studies. Although not formally tested, few differences were identified between the populations of European $v$. Australian adolescents: energy intake was higher among Australian adolescents whereas the degree of under-reporting of energy intake was higher in European adolescents. Furthermore, screen time use was higher among European adolescents, whereas MVPA was higher among Australian adolescents. The prevalence of OW/obesity was higher in Australia. Sex differences were very similar in both studies: energy intake and DED were higher in boys whereas under-reporting was higher in girls; fibre intake was lower and DP scores were higher in boys; MVPA and screen time were higher in boys. However, OW and obesity prevalence was higher in European boys (HELENA study) only.

\section{Dietary pattern scores in relation to diet}

Table 2 shows the derived dietary pattern including the factor loadings of all food groups. The dietary pattern scores were highly correlated with DED ( $r 0.68$ and 0.73 for HELENA and NCNPAS, respectively) and inversely with FD $(r-0.59$ and -0.65 for HELENA and NCNPAS, respectively) and low to moderately correlated with percentage of energy intake from fat ( $r 0.15$ and 0.32 for HELENA and NCNPAS, respectively). Factor loadings from both surveys indicated that a high pattern score was associated with higher consumption of biscuits and cakes, 
Table 1. Description of the adolescent population included in the analyses, stratified for the Healthy Lifestyle in Europe by Nutrition in Adolescence study (HELENA) and the Australian National Children's Nutrition and Physical Activity Survey (NCNPAS)

(Percentages and 95\% confidence intervals; mean values and standard deviations; medians and interquartile ranges)

\begin{tabular}{|c|c|c|c|c|c|c|c|c|c|c|c|c|c|c|}
\hline & \multicolumn{7}{|c|}{ HELENA } & \multicolumn{7}{|c|}{ NCNPAS } \\
\hline & \multicolumn{2}{|c|}{ All $(n$ 1954) } & \multicolumn{2}{|c|}{ Boys $(n 886)$} & \multicolumn{2}{|c|}{ Girls ( $n$ 1068) } & \multirow[b]{2}{*}{$P$} & \multicolumn{2}{|c|}{ All $(n$ 1498) } & \multicolumn{2}{|c|}{ Boys ( $n$ 742) } & \multicolumn{2}{|c|}{ Girls $(n 756)$} & \multirow[b]{2}{*}{$P$} \\
\hline & $\%$ & $95 \% \mathrm{Cl}$ & $\%$ & $95 \% \mathrm{Cl}$ & $\%$ & $95 \% \mathrm{Cl}$ & & $\%$ & $95 \% \mathrm{Cl}$ & $\%$ & $95 \% \mathrm{Cl}$ & $\%$ & $95 \% \mathrm{Cl}$ & \\
\hline Age (years) & & & & & & & 0.28 & & & & & & & 0.23 \\
\hline Mean & & 14.6 & & 14.6 & & 14.6 & & & $14 \cdot 2$ & & $14 \cdot 2$ & & $14 \cdot 15$ & \\
\hline SD & & $1 \cdot 2$ & & 1.2 & & 1.2 & & & 1.3 & & 1.3 & & 1.31 & \\
\hline \multicolumn{15}{|l|}{ Maternal education level } \\
\hline Low & 31.5 & $29 \cdot 5,33.6$ & $32 \cdot 4$ & $29 \cdot 3,35.5$ & 30.8 & $28 \cdot 0,33 \cdot 6$ & & $30 \cdot 4$ & $28 \cdot 1,32 \cdot 8$ & 31.8 & $28 \cdot 4,35 \cdot 2$ & $29 \cdot 1$ & $25 \cdot 9,32 \cdot 3$ & \\
\hline Intermediate & $31 \cdot 1$ & $29 \cdot 0,33 \cdot 1$ & 29.5 & $26 \cdot 4,32.5$ & 32.4 & $29 \cdot 6,35 \cdot 2$ & & 39.1 & $36 \cdot 6,41 \cdot 6$ & $36 \cdot 2$ & $32 \cdot 8,39 \cdot 7$ & 41.9 & $38 \cdot 4,45 \cdot 4$ & \\
\hline High & 37.4 & $35 \cdot 3,39 \cdot 6$ & $38 \cdot 1$ & $34.9,41 \cdot 3$ & 36.8 & $33 \cdot 9,36 \cdot 7$ & 0.37 & 30.4 & $28 \cdot 1,32 \cdot 8$ & 31.9 & $28 \cdot 6,35 \cdot 3$ & 29.0 & $25 \cdot 7,32 \cdot 2$ & 0.079 \\
\hline Energy intake (kJ/d) & & & & & & & $<0.0001$ & & & & & & & $<0.0001$ \\
\hline Mean & \multicolumn{2}{|c|}{9081.9} & \multicolumn{2}{|c|}{$10470 \cdot 6$} & \multicolumn{2}{|r|}{$7929 \cdot 8$} & & \multicolumn{2}{|r|}{$9488 \cdot 9$} & \multicolumn{2}{|c|}{$10821 \cdot 3$} & \multicolumn{2}{|r|}{$8181 \cdot 2$} & \\
\hline SD & \multirow{2}{*}{\multicolumn{2}{|c|}{$3281 \cdot 7$}} & \multicolumn{2}{|c|}{3617.9} & \multirow{2}{*}{\multicolumn{2}{|c|}{$2434 \cdot 3$}} & & \multirow{2}{*}{\multicolumn{2}{|c|}{$3025 \cdot 2$}} & \multirow{2}{*}{\multicolumn{2}{|c|}{$3104 \cdot 6$}} & & $2286 \cdot 6$ & \\
\hline Fibre intake (mg/kJ) & & & & & & & $<0.0001$ & & & & & & & $<0.0001$ \\
\hline Mean & & $2 \cdot 1$ & & 1.9 & & $2 \cdot 2$ & & & 2.5 & & $2 \cdot 4$ & & $2 \cdot 6$ & \\
\hline SD & & 0.8 & & 0.7 & & 0.8 & & & 0.8 & & 0.7 & & 0.8 & \\
\hline Fat contribution to energy intake (\%) & & & & & & & 0.58 & & & & & & & 0.41 \\
\hline Mean & & 33.8 & & $33 \cdot 7$ & & 33.9 & & & $31 \cdot 1$ & & $31 \cdot 3$ & & 31.0 & \\
\hline SD & & 8.4 & & 8.5 & & 8.4 & & & 0.1 & & 5.5 & & $6 \cdot 0$ & \\
\hline Energy density of food intake $(\mathrm{kJ} / \mathrm{g})$ & & & & & & & $<0.0001$ & & & & & & & $<0.0001$ \\
\hline Mean & & 7.9 & & $8 \cdot 2$ & & 7.6 & & & 7.9 & & $8 \cdot 1$ & & 7.6 & \\
\hline SD & & 1.9 & & 2.0 & & 1.9 & & & 1.7 & & 1.6 & & 1.7 & \\
\hline Dietary pattern score & & & & & & & $<0.0001$ & & & & & & & $<0.0001$ \\
\hline Mean & & -0.0 & & 0.2 & & -0.2 & & & 0.0 & & 0.2 & & $-0 \cdot 2$ & \\
\hline SD & & 1.0 & & 1.1 & & 0.9 & & & 1.2 & & 1.3 & & $1 \cdot 1$ & \\
\hline Energy under-reporting status (under-reporters) & 21.5 & $19 \cdot 7,23 \cdot 3$ & 19.5 & $16 \cdot 9,22 \cdot 1$ & $23 \cdot 1$ & $20 \cdot 6,25 \cdot 7$ & 0.054 & $12 \cdot 7$ & $11 \cdot 1,14 \cdot 4$ & 9.6 & $7 \cdot 4,11 \cdot 7$ & $15 \cdot 9$ & $13 \cdot 3,18 \cdot 5$ & $<0.0001$ \\
\hline Screen time $(\min / \mathrm{d})$ & & & & & & & $<0.0001$ & & & & & & & $<0.0001$ \\
\hline Median & & $242 \cdot 1$ & & $286 \cdot 1$ & & $214 \cdot 3$ & & & 227.5 & & $258 \cdot 7$ & & $196 \cdot 2$ & \\
\hline Interquartiles & 160 & $.7-368.6$ & & $3.4-398.6$ & & $5.8-330.0$ & & & $3 \cdot 2-306 \cdot 2$ & & $3.7-338.7$ & & $1.9-276.9$ & \\
\hline MVPA (min/d) & & & & & & & $<0.0001$ & & & & & & & $<0.0001$ \\
\hline Median & & 81.4 & & 96.4 & & $70 \cdot 0$ & & & 97.5 & & $118 \cdot 1$ & & 79.4 & \\
\hline Interquartiles & & $.0-150 \cdot 7$ & & $.4-171.4$ & & $.0-132 \cdot 9$ & & & $.2-148.7$ & & $.0-170.0$ & & $0-126 \cdot 2$ & \\
\hline Meeting MVPA recommandations & $62 \cdot 1$ & $59 \cdot 9,64 \cdot 2$ & 68.6 & $65 \cdot 6,71 \cdot 7$ & 56.6 & $53 \cdot 7,59 \cdot 6$ & $<0.0001$ & 73.8 & $71 \cdot 6,76 \cdot 1$ & 82.2 & $79 \cdot 4,85 \cdot 0$ & 65.6 & $62 \cdot 2,69 \cdot 0$ & $<0.0001$ \\
\hline Weight status & & & & & & & & & & & & & & \\
\hline Normal weight & 79.0 & $77 \cdot 2,80 \cdot 8$ & 76.5 & $73 \cdot 7,79 \cdot 3$ & $81 \cdot 1$ & $78 \cdot 7,83 \cdot 4$ & & $75 \cdot 7$ & $73 \cdot 4,77 \cdot 7$ & $76 \cdot 0$ & $72 \cdot 9,79 \cdot 1$ & $75 \cdot 1$ & $72 \cdot 0,78 \cdot 2$ & \\
\hline OW & $15 \cdot 8$ & $14 \cdot 2,17 \cdot 4$ & $16 \cdot 6$ & $14 \cdot 1,19 \cdot 0$ & $15 \cdot 2$ & $13 \cdot 0,17 \cdot 3$ & & 18.4 & $16 \cdot 4,20 \cdot 3$ & 18.6 & $15 \cdot 8,21 \cdot 4$ & $18 \cdot 1$ & $15 \cdot 4,20 \cdot 9$ & \\
\hline Obese & $5 \cdot 2$ & $4 \cdot 2,6 \cdot 1$ & 6.9 & $5 \cdot 2,8 \cdot 6$ & 3.7 & $2 \cdot 6,4.9$ & 0.004 & $6 \cdot 1$ & $4 \cdot 9,7 \cdot 3$ & 5.4 & $3 \cdot 8,7 \cdot 0$ & 6.7 & $5 \cdot 0,8 \cdot 5$ & 0.54 \\
\hline Body fat (\%) & & & & & & & $<0.0001$ & & & & & & & \\
\hline Mean & & $22 \cdot 7$ & & $19 \cdot 1$ & & $25 \cdot 7$ & & & & & & & & \\
\hline SD & & $10 \cdot 2$ & & 11.8 & & 7.5 & & & & & & & & \\
\hline
\end{tabular}

MVPA, moderate to vigorous physical activity; OW, overweight. 
Table 2. Reduced rank regression factor loadings for the dietary patterns derived from the dietary intake assessments in European (Healthy Lifestyle in Europe by Nutrition in Adolescence (HELENA)) and Australian (National Children's Nutrition and Physical Activity Survey (NCNPAS)) adolescents

\begin{tabular}{|c|c|c|}
\hline & HELENA & NCNPAS \\
\hline High-fat milk and cream & 0.018 & $0 \cdot 151$ \\
\hline Low-fat milk & -0.028 & -0.108 \\
\hline Yogurts & $-0.215 \dagger$ & $-0.152 \dagger$ \\
\hline Cheese & 0.141 & 0.114 \\
\hline Butter and animal fat & 0.139 & 0.118 \\
\hline Margarine and vegetable oils & $0 \cdot 107$ & 0.081 \\
\hline Egg and egg dishes & 0.037 & 0.081 \\
\hline Low-fibre bread & 0.135 & $0.177 \dagger$ \\
\hline High-fibre bread & $-0.178 \dagger$ & $-0.216 \dagger$ \\
\hline Other bread products & 0.093 & 0.054 \\
\hline High-fibre breakfast cereals & 0.016 & $-0.156 \dagger$ \\
\hline Other breakfast cereals & $-0 \cdot 101$ & 0.001 \\
\hline Rice, pasta and other grains & -0.019 & $-0.166 \dagger$ \\
\hline Cereal-based mixed meals & & -0.018 \\
\hline Pizza & & 0.095 \\
\hline Biscuits and cakes & $0.204 \dagger$ & $0.159 \dagger$ \\
\hline Puddings & 0.066 & 0.032 \\
\hline Ice creams & 0.050 & 0.079 \\
\hline Chocolate and confectionery & $0.237 \dagger$ & $0.216 \dagger$ \\
\hline Spreads & 0.001 & -0.045 \\
\hline Meat and poultry & $0 \cdot 120$ & -0.011 \\
\hline Meat-mixed dishes & & 0.090 \\
\hline Processed meat & 0.142 & $0.175 \dagger$ \\
\hline Coated or breaded meat and fish & 0.036 & $0.151 \dagger$ \\
\hline Meat substitutes & $-0 \cdot 116$ & -0.069 \\
\hline Fish & -0.004 & -0.052 \\
\hline Fried or roast potatoes & 0.057 & $0.206 \dagger$ \\
\hline Boiled or baked potatoes & -0.003 & -0.128 \\
\hline Vegetables & $-0.427 \dagger$ & $-0.354 \dagger$ \\
\hline Legumes & $-0.177 \dagger$ & -0.125 \\
\hline Vegetable mixed dishes & & -0.049 \\
\hline Fresh fruits & $-0.570 \dagger$ & $-0.474 \dagger$ \\
\hline Other fruits & -0.054 & $-0 \cdot 102$ \\
\hline Nuts and seeds & -0.020 & -0.035 \\
\hline Crisps and savoury snacks & $0.180 \dagger$ & $0.197 \dagger$ \\
\hline Soups & -0.128 & $-0.216 \dagger$ \\
\hline Sauces (low energy dense) & -0.012 & -0.084 \\
\hline Sauces (high energy dense) & 0.126 & -0.003 \\
\hline Condiments & & -0.055 \\
\hline Sugar-sweetened beverages & $0.210 \dagger$ & $0.198 \dagger$ \\
\hline Low-energy beverages & 0.060 & 0.062 \\
\hline Fruit juice & 0.042 & -0.052 \\
\hline Hot and powdered drinks & -0.051 & 0.001 \\
\hline Water & $-0 \cdot 108$ & $-0.176 \dagger$ \\
\hline Percentage contribution to the total inertia & 30.5 & $46 \cdot 6$ \\
\hline Correlation with DED & $0.68^{\star}$ & $0.73^{\star}$ \\
\hline Correlation with FD & $-0.59^{*}$ & $-0.65^{\star}$ \\
\hline Correlation with percentage of energy from fat & $0.15^{\star}$ & $0.32^{*}$ \\
\hline
\end{tabular}

$\mathrm{DED}$, dietary energy density; FD, fibre density.

${ }^{*} P<0.0001$.

$\dagger$ Factor loadings $>0.15$.

chocolate and confectionery, crisps and savoury snacks, sugar-sweetened beverages, and lower consumption of yogurt, high-fibre bread, vegetables, fresh fruit. In NCNPAS, a high pattern score was additionally associated with higher consumption of full-fat milk and cream, low-fibre bread, processed meat, fried potatoes, coated or breaded meat and fish and lower consumption of breakfast cereals, rice, pasta and other grains, soups and water as a beverage whereas a high pattern score was associated with a lower consumption of legumes in HELENA.

\section{Dietary pattern scores in relation to social and lifestyle factors (potential confounders)}

Dietary pattern scores were found to be positively associated with screen time (in both studies), whereas an inverse association was observed with meeting PA recommendations (in Europe only) (Table 3). Significant inverse associations were also found with maternal education in both data sets. No associations were found between the dietary pattern scores and energy intake or energy under-reporting among the Australian adolescents. However, among European adolescents a strong inverse association was found between the dietary pattern scores and under-reporting, and a positive association between the dietary pattern scores and energy intake.

\section{Dietary pattern scores in relation to adolescents' BMI and body weight percentage}

The multivariable regression analysis indicated that dietary pattern scores were inversely associated with BMI $z$-scores in Australian adolescent boys and borderline in European adolescent boys (Table 4). This inverse association was also borderline significant for \%BF among European boys. Similarly, a lower likelihood for OW in boys was observed with higher DP scores in both surveys (though only significant in Australian boys $P=0.03$ ). No such relationships were observed in adolescent girls in either data sets.

\section{Social and lifestyle factors in relation to adolescents' $B M I$ and body fat percentage}

Maternal education level was inversely associated with any of the outcomes used (BMI $z$-scores, OW and \%BF). Under-reporting was positively associated with all anthropometric outcomes and more in particular among boys, especially for \%BF.

Among European girls, screen time was found to be positively associated with \%BF only. In European boys, meeting MVPA recommendations was inversely associated with \%BF. Although screen time was also positively associated with BMI $z$-scores and a higher risk for OW in Australian boys, no significant relationships were found with PA in the Australian data set.

There was no significant interaction between dietary pattern scores and under-reporting status in the multivariable analyses addressing the anthropometric measurements as dependent variables (Table 4), so therefore we did not stratify our analyses by under-reporting status. Besides, the factors loadings were similar across normal- and under-reporters (results not shown). Further adjustment for energy intake in the multivariable models hardly changed the associations between dietary pattern scores and any of BMI $z$-scores, OW and \%BF (results not shown).

\section{Discussion}

This study aimed at comparing DP derived using RRR with energy density, percentage of energy intake from fat and FD as the intermediate variables from two wide-scale samples with 
Table 3. Results from the multivariable linear regression analyses, with the energy-dense, low-fibre, high-fat dietary pattern as the dependent variable and lifestyle (dietary patterns scores, meeting physical activity (PA) recommendations, screen sedentary behaviour, energy under-reporting status) and socio-economic factors as the independent variables of interest in European (Healthy Lifestyle in Europe by Nutrition in Adolescence (HELENA)) and Australian (National Children's Nutrition and Physical Activity Survey (NCNPAS)) adolescents, respectively

(Linear regression coefficients and $95 \%$ confidence intervals)

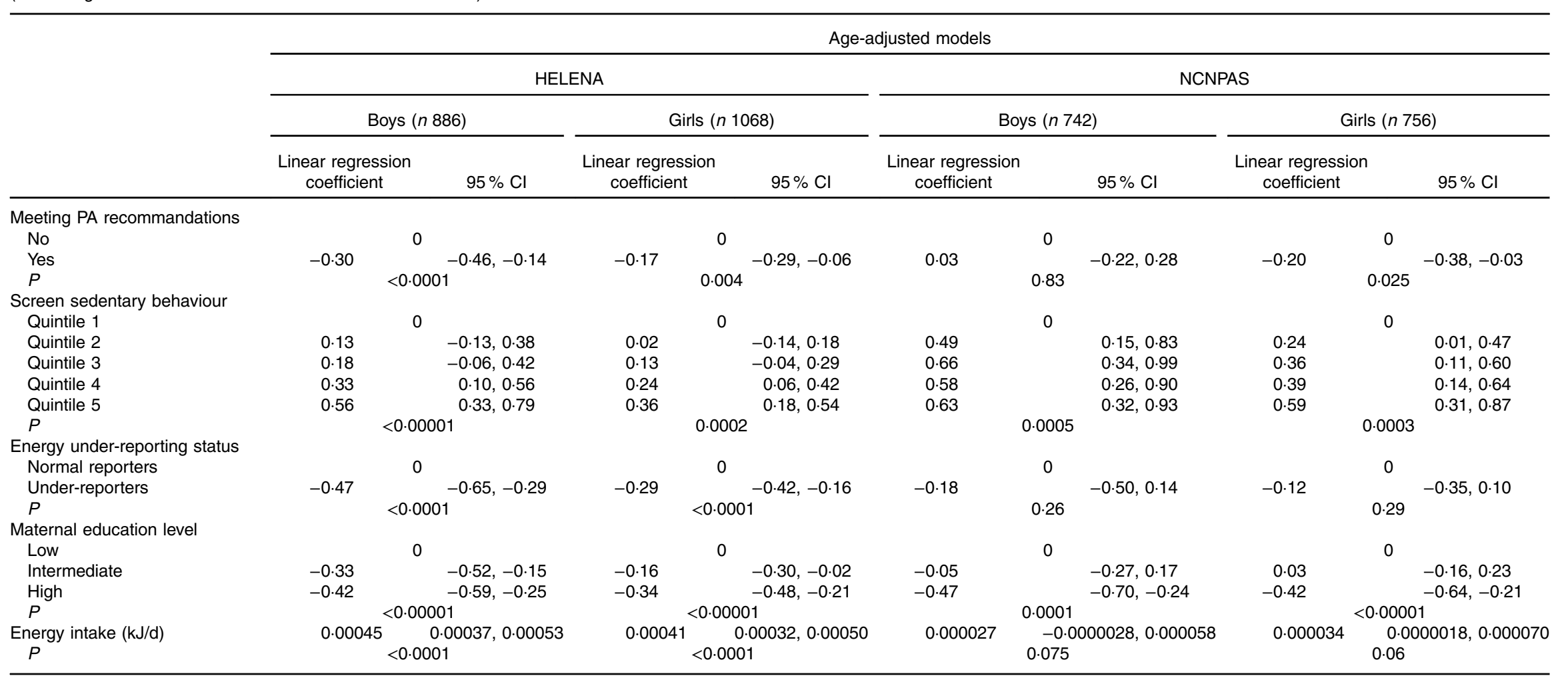


Table 4. Results from the multivariable linear and logistic regression analyses, with BMI $z$-scores, body fat percentage (\%BF) and overweight (OW) as the dependent variables and lifestyle (dietary patterns scores, meeting physical activity (PA) recommendations, screen sedentary behaviour, energy under-reporting status) and socio-economic factors as the independent variables of interest in European (Healthy Lifestyle in Europe by Nutrition in Adolescence (HELENA)) and Australian (National Children's Nutrition and Physical Activity Survey (NCNPAS)) adolescents, respectively

$(\beta$ Values and standard deviations; odds ratios $(\mathrm{OR})$ and $95 \%$ confidence intervals)

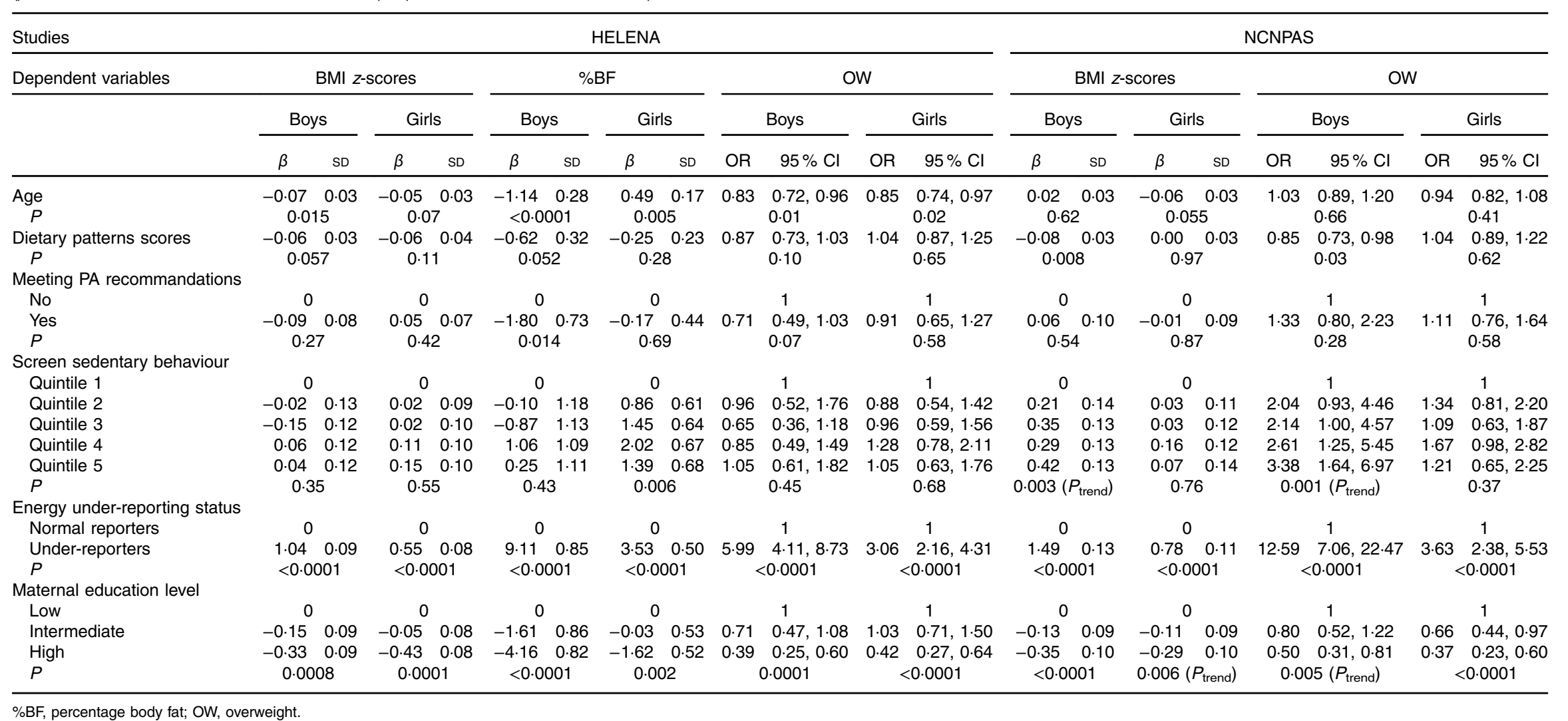

\%BF, percentage body fat; OW, overweight. 
different geographical, cultural and socio-economic characteristics. The DP identified in European and Australian adolescents exhibited similarities in their food characterisation and were also consistent with the 'energy-dense, high-fat, low-fibre' dietary pattern found in both the ALSPAC study from Avon (UK) ${ }^{(13)}$ and the Raine Study from Perth (Western Australia $)^{(11,12)}$. In the later, a similar dietary pattern was obtained using two different tools to assess dietary intakes, that is a FFQ and a 3 -d food record ${ }^{(11)}$. Overall, high scores on this pattern indicated adherence to a diet with a low consumption of fresh fruit and vegetables and a high consumption of crisps and snacks, chocolate and confectionery ${ }^{(11-13,20)}$. This suggests that when using nutrient intakes as the intermediate markers for obesity and similar food groupings, the resulting RRR DP can be replicated in other study samples of adolescents from Western environments.

Analyses investigating associations between the 'energydense, high-fat, low-fibre' dietary pattern scores and covariates confirmed previous findings that this dietary pattern was inversely associated with the educational level of the mother and positively associated with screen time. For example, a high maternal education was considered as a positive influencing factor for a healthy food intake, especially in childhood ${ }^{(44)}$, and also in adolescents ${ }^{(45,46)}$. Previous studies also identified associations between low SES, using family income parameters instead of education of the mother, and 'unhealthy dietary pattern' in developed countries ${ }^{(45-49)}$. Therefore, considering that we identified associations consistent with previous literature regarding the DP and SES and lifestyle factors, it suggests that the inverse associations between the dietary pattern and adiposity/OW in adolescent boys and the absence of significant association in girls was not due to poor data quality. Rather, this association is more likely explained by the cross-sectional design of the HELENA and NCNPAS studies. Indeed, the review by Ambrosini $^{(6)}$, which included studies with longitudinal design only, provides evidence for a prospective and positive association between empirically derived DP in childhood and adolescence that are high in energy-dense, high-fat, low-fibre foods and later obesity risk.

These contradictory regression results found in our study $v$. those earlier published from the ALSPAC study (positive association between the same dietary pattern and FM index in children) ${ }^{(13)}$ may also partly be due to increased misreporting with increasing age. In the follow-up of the ALSPAC study, a decline could be seen in the association between the dietary pattern scores and later adiposity when comparing the children at the age of $7 v .13$ years old ${ }^{(20)}$. The authors suggested that this might be due to increasing levels of dietary measurement error with age of the child as the prevalence of dietary underreporting increased with age and was most prevalent at 13 years of age. Further, given that body image becomes more important with age, adolescents are more likely to follow a restrictive diet as compared with their younger counterparts in the presence of obesity, possibly attenuating any associations between obesity and energy-dense diets in cross-sectional studies ${ }^{(50)}$. In fact, another important result was that the energy under-reporters scored low in the 'energy-dense, high-fat, low-fibre' dietary pattern and were also more likely to experience excessive weight, in particular boys (especially for FM). Therefore underreporting of energy-dense, high-fat, low-fibre foods due to social desirable answers among the obese and/or true underconsumption of these specific foods in order to lose weight $^{(39,50)}$, could have contributed to reversing the crosssectional association under study, especially in boys.

The strengths of the study include the use of two adolescent wide-scale samples of Europe and Australia (part of two continents with different geographical, cultural and socio-economic characteristics), respectively, using similar assessment methods and measurements and the large number of adolescents included in both surveys. Few studies so far have used populationbased surveys with large sample size to identify DP in adolescents $^{(47)}$. Also, both studies have similar study aims and used comparable methods to assess dietary intake. Furthermore, RRR analysis demonstrated a similar DP in European and Australian adolescents that was also consistent with the 'energydense, high-fat, low-fibre' DP obtained in the ALSPAC study, as well as with the one derived in the Raine study ${ }^{(11,12)}$. Finally, both studies included anthropometric, SES and lifestyle data (including PA and SB) obtained via standardised procedures.

Some limitations of the analysis should be considered. We used two cross-sectional studies, which did not allow the assessment of temporal relations and thus neither causal inference. Differences in food habits and culinary use between Europe and Australia and in food composition tables should be acknowledged. However, we harmonised the food groupings as far as possible using the same nutritional composition criteria, in accordance with the ALSPAC classification. In addition, while the two studies were comparable with respect to some covariates (e.g. maternal education level), there were slight differences regarding the definition of $\mathrm{PA}$ and $\mathrm{SB}$. In particular, the more detailed categories of behaviour for sedentary time questions in HELENA $v$. NCNPAS might have tended to over-estimate the duration and be partly responsible for the higher sedentary time found in HELENA. Possible differences in the study protocol for dietary intake assessments between HELENA and NCNPAS (self-reported $v$. interview based) might also have contributed to the higher level of under-reporting in the HELENA study. These differences in study protocols might partly explain the minor differences found in the associations with the 'energy-dense, high-fat, low-fibre' dietary pattern between the European and Australian adolescents samples.

In conclusion, the DP identified in these Australian and European studies were very similar and comparable to those identified in a previous study of DP using RRR along with energy density, FD and percentage of energy intake from fat as the intermediate variables. This demonstrates the generalisability and robustness of the RRR method in calculating DP among populations. However, this cross-country study confirms that when studying diet-obesity relationships in adolescents, longitudinal designs are more relevant to prevent reverse causality.

\section{Acknowledgements}

The authors acknowledge all the adolescents who made the HELENA study possible and the families participating in the NCNPAS study. 
The HELENA study received funding from the European Union's Sixth RTD Framework Programme (contracts FOODCT-2007-036196-2 and FOODCT-2005-007034, respectively). Additional support was obtained from the Spanish Ministry of Education (AGL2007-29784-E/ALI). S. A. M. is supported by an NHMRC Career Development Fellowship Level 2, ID1104636 and was previously supported by an ARC Future Fellowship (2011-2015, FT100100581). S. L. is supported by a French National Research Agency (grant no. ANR-12-DSSA-0001) and was previously supported by an Alfred Deakin postdoctoral Fellowship (Deakin University, 2010-2012). None of the funders had any role in the design, analysis or writing of this article.

I. H. and S. L. contributed to the designed research, analysed data, performed statistical analysis and wrote the paper; S. M. contributed to the design of the analysis, advised on the dietary analysis of the NCNPAS data, assisted with the interpretation of data analysis and critically revised the draft manuscript. T. M. contributed with analysed data and critically revised the paper drafts. L. M. and the rest of the authors contributed to the HELENA study data collection and handling. All authors have read and approved the final manuscript.

None of the authors has declared conflicts of interest.

\section{References}

1. Wang H, Dwyer-Lindgren L, Lofgren KT, et al. (2012) Age-specific and sex-specific mortality in 187 countries, 1970-2010: a systematic analysis for the Global Burden of Disease Study 2010. Lancet 380, 2071-2094.

2. Bauer UE, Briss PA, Goodman RA, et al. (2014) Prevention of chronic disease in the 21st century: elimination of the leading preventable causes of premature death and disability in the USA. Lancet 384, 45-52.

3. Hu FB (2002) Dietary pattern analysis: a new direction in nutritional epidemiology. Curr Opin Lipidol 13, 3-9.

4. Hoffmann K, Schulze MB, Schienkiewitz A, et al. (2004) Application of a new statistical method to derive dietary patterns in nutritional epidemiology. Am J Epidemiol 159, 935-944.

5. Gleason PM, Boushey CJ, Harris JE, et al. (2015) Publishing nutrition research: a review of multivariate techniquespart 3: data reduction methods. I Acad Nutr Diet 115 , 1072-1082.

6. Ambrosini GL (2014) Childhood dietary patterns and later obesity: a review of the evidence. Proc Nutr Soc 73, 137-146.

7. Ocke MC (2013) Evaluation of methodologies for assessing the overall diet: dietary quality scores and dietary pattern analysis. Proc Nutr Soc 72, 191-199.

8. Kant AK (2004) Dietary patterns and health outcomes. J Am Diet Assoc 104, 615-635.

9. Newby PK \& Tucker KL (2004) Empirically derived eating patterns using factor or cluster analysis: a review. Nutr Rev 62 , $177-203$.

10. Manios Y, Kourlaba G, Grammatikaki E, et al. (2010) Comparison of two methods for identifying dietary patterns associated with obesity in preschool children: the GENESIS study. Eur J Clin Nutr 64, 1407-1414.

11. Appannah G, Pot GK, O'Sullivan TA, et al. (2014) The reliability of an adolescent dietary pattern identified using reduced-rank regression: comparison of a FFQ and $3 \mathrm{~d}$ food record. Br J Nutr 112, 609-615.
12. Appannah G, Pot GK, Huang RC, et al. (2015) Identification of a dietary pattern associated with greater cardiometabolic risk in adolescence. Nutr Metab Cardiovasc Dis 25, 643-650.

13. Johnson L, Mander AP, Jones LR, et al. (2008) Energy-dense, low-fiber, high-fat dietary pattern is associated with increased fatness in childhood. Am J Clin Nutr 87, 846-854.

14. Hoffmann K, Zyriax BC, Boeing $\mathrm{H}$, et al. (2004) A dietary pattern derived to explain biomarker variation is strongly associated with the risk of coronary artery disease. Am J Clin Nutr 80, 633-640.

15. Hoffmann K, Boeing H, Boffetta $\mathrm{P}$, et al. (2005) Comparison of two statistical approaches to predict all-cause mortality by dietary patterns in German elderly subjects. Br J Nutr $\mathbf{9 3}$, 709-716.

16. McCann SE, McCann WE, Hong CC, et al. (2007) Dietary patterns related to glycemic index and load and risk of premenopausal and postmenopausal breast cancer in the Western New York Exposure and Breast Cancer Study. Am J Clin Nutr 86, 465-471.

17. Nettleton JA, Steffen LM, Schulze MB, et al. (2007) Associations between markers of subclinical atherosclerosis and dietary patterns derived by principal components analysis and reduced rank regression in the Multi-Ethnic Study of Atherosclerosis (MESA). Am J Clin Nutr 85, 1615-1625.

18. Schulz M, Nothlings U, Hoffmann K, et al. (2005) Identification of a food pattern characterized by high-fiber and low-fat food choices associated with low prospective weight change in the EPIC-Potsdam cohort. J Nutr 135, 1183-1189.

19. Schulze MB, Hoffmann K, Manson JE, et al. (2005) Dietary pattern, inflammation, and incidence of type 2 diabetes in women. Am J Clin Nutr 82, 675-684.

20. Ambrosini GL, Emmett PM, Northstone K, et al. (2012) Identification of a dietary pattern prospectively associated with increased adiposity during childhood and adolescence. Int $J$ Obes (Lond) 36, 1299-1305.

21. Imamura F, Lichtenstein AH, Dallal GE, et al. (2009) Generalizability of dietary patterns associated with incidence of type 2 diabetes mellitus. Am J Clin Nutr 90, 1075-1083.

22. Moreno LA, De HS, Gonzalez-Gross M, et al. (2008) Design and implementation of the Healthy Lifestyle in Europe by Nutrition in Adolescence Cross-Sectional Study. Int J Obes (Lond) 32, Suppl. 5, S4-S11.

23. Vereecken CA, Covents M, Matthys C, et al. (2005) Young adolescents' nutrition assessment on computer (YANA-C). Eur J Clin Nutr 59, 658-667.

24. Vereecken CA, Covents M, Sichert-Hellert W, et al. (2008) Development and evaluation of a self-administered computerized 24-h dietary recall method for adolescents in Europe. Int J Obes (Lond) 32, Suppl. 5, S26-S34.

25. Biro G, Hulshof KF, Ovesen L, et al. (2002) Selection of methodology to assess food intake. Eur J Clin Nutr $\mathbf{5 6}$, Suppl. 2, S25-S32.

26. De Cocker K, Ottevaere C, Sjostrom M, et al. (2011) Self-reported physical activity in European adolescents: results from the HELENA (Healthy Lifestyle in Europe by Nutrition in Adolescence) study. Public Health Nutr 14, 246-254.

27. Hagstromer M, Bergman P, De Bourdeaudhuij I, et al. (2008) Concurrent validity of a modified version of the International Physical Activity Questionnaire (IPAQ-A) in European adolescents: The HELENA Study. Int J Obes (Lond) 32, Suppl. 5, S42-S48.

28. World Health Organization (2010) Global Recommendations on Physical Activity for Health. Geneva: WHO.

29. Rey-Lopez JP, Vicente-Rodriguez G, Ortega FB, et al. (2010) Sedentary patterns and media availability in European adolescents: the HELENA study. Prev Med 51, 50-55. 
30. Nagy E, Vicente-Rodriguez G, Manios Y, et al. (2008) Harmonization process and reliability assessment of anthropometric measurements in a multicenter study in adolescents. Int J Obes (Lond) 32, Suppl. 5, S58-S65.

31. Cole TJ \& Lobstein T (2012) Extended international (IOTF) body mass index cut-offs for thinness, overweight and obesity. Pediatr Obes 7, 284-294.

32. Cole TJ, Freeman JV \& Preece MA (1998) British 1990 growth reference centiles for weight, height, body mass index and head circumference fitted by maximum penalized likelihood. Stat Med 17, 407-429.

33. Slaughter MH, Lohman TG, Boileau RA, et al. (1988) Skinfold equations for estimation of body fatness in children and youth. Hum Biol 60, 709-723.

34. Department of Health and Ageing (2007) User guide - 2007 Australian National Children's Nutrition and Physical Activity Survey. http://www.health.gov.au/internet/main/publishing. nsf/Content/phd-nutrition-childrens-survey-userguide (accessed August 2016).

35. Food Standards Australia New Zealand (2008) AUSNUT 2007. Australian Food, Supplement and Nutrient Database for Estimation of Population Nutrient Intakes. Canberra: Food Standards Australia New Zealand.

36. Ridley K, Olds TS \& Hill A (2006) The Multimedia Activity Recall for Children and Adolescents (MARCA): development and evaluation. Int J Behav Nutr Phys Act 3, 10.

37. Commonwealth Scientific Industrial Research Organisation (CSIRO) (2008) 2007 Australian National Children's Nutrition and Physical Activity Survey. Canberra: Department of Health and Ageing.

38. Rennie KL, Coward A \& Jebb SA (2007) Estimating underreporting of energy intake in dietary surveys using an individualised method. Br J Nutr 97, 1169-1176.

39. Lioret S, Touvier M, Balin M, et al. (2011) Characteristics of energy under-reporting in children and adolescents. Br J Nutr 105, 1671-1680.

40. Schofield WN (1985) Predicting basal metabolic rate, new standards and review of previous work. Hum Nutr Clin Nutr 39, 5-41.

41. Black AE (2000) Critical evaluation of energy intake using the Goldberg cut-off for energy intake:basal metabolic rate.
A practical guide to its calculation, use and limitations. Int J Obes Relat Metab Disord 24, 1119-1130.

42. Livingstone MB, Robson PJ, Black AE, et al. (2003) An evaluation of the sensitivity and specificity of energy expenditure measured by heart rate and the Goldberg cut-off for energy intake: basal metabolic rate for identifying mis-reporting of energy intake by adults and children: a retrospective analysis. Eur J Clin Nutr 57, 455-463.

43. Schulze MB, Hoffmann K, Kroke A, et al. (2003) An approach to construct simplified measures of dietary patterns from exploratory factor analysis. Br J Nutr 89, 409-419.

44. van Ansem WJ, Schrijvers CT, Rodenburg G, et al. (2014) Maternal educational level and children's healthy eating behaviour: role of the home food environment (crosssectional results from the INPACT study). Int J Behav Nutr Phys Act 11, 113.

45. Northstone K, Smith AD, Cribb VL, et al. (2014) Dietary patterns in UK adolescents obtained from a dual-source FFQ and their associations with socio-economic position, nutrient intake and modes of eating. Public Health Nutr 17, 1476-1485.

46. Aranceta J, Perez-Rodrigo C, Ribas L, et al. (2003) Sociodemographic and lifestyle determinants of food patterns in Spanish children and adolescents: the enKid study. Eur J Clin Nutr 57, Suppl. 1, S40-S44.

47. Craig LC, McNeill G, Macdiarmid JI, et al. (2010) Dietary patterns of school-age children in Scotland: association with socio-economic indicators, physical activity and obesity. $\mathrm{BrJ}$ Nutr 103, 319-334.

48. Richter A, Heidemann C, Schulze MB, et al. (2012) Dietary patterns of adolescents in Germany - associations with nutrient intake and other health related lifestyle characteristics. BMC Pediatr 12, 12-35.

49. Kourlaba G, Panagiotakos DB, Mihas K, et al. (2009) Dietary patterns in relation to socio-economic and lifestyle characteristics among Greek adolescents: a multivariate analysis. Public Health Nutr 12, 1366-1372.

50. Bel-Serrat S, Julian-Almarcegui C, Gonzalez-Gross $\mathrm{M}$, et al. (2016) Correlates of dietary energy misreporting among European adolescents: the Healthy Lifestyle in Europe by Nutrition in Adolescence (HELENA) study. Br J Nutr $\mathbf{1 1 5}$, 1439-1452. 